\title{
Inter-hemispheric temperature variability over the past millennium
}

\author{
Raphael Neukom ${ }^{1,2 \star \dagger}$, Joëlle Gergis ${ }^{3}$, David J. Karoly ${ }^{3,4}$, Heinz Wanner ${ }^{1}$, Mark Curran ${ }^{5,6}$, Julie Elbert' \\ Fidel González-Rouco ${ }^{7}$, Braddock K. Linsley ${ }^{8}$, Andrew D. Moy 5,6, Ignacio Mundo9,10, \\ Christoph C. Raible ${ }^{1,11}$, Eric J. Steig ${ }^{12}$, Tas van Ommen ${ }^{5,6}$, Tessa Vance ${ }^{6}$, Ricardo Villalba ${ }^{9}$, \\ Jens Zinke ${ }^{13,14}$ and David Frank ${ }^{1,2}$
}

The Earth's climate system is driven by a complex interplay of internal chaotic dynamics and natural and anthropogenic external forcing. Recent instrumental data have shown a remarkable degree of asynchronicity between Northern Hemisphere and Southern Hemisphere temperature fluctuations, thereby questioning the relative importance of internal versus external drivers of past as well as future climate variability ${ }^{1-3}$. However, large-scale temperature reconstructions for the past millennium have focused on the Northern Hemisphere ${ }^{4,5}$, limiting empirical assessments of inter-hemispheric variability on multi-decadal to centennial timescales. Here, we introduce a new millennial ensemble reconstruction of annually resolved temperature variations for the Southern Hemisphere based on an unprecedented network of terrestrial and oceanic palaeoclimate proxy records. In conjunction with an independent Northern Hemisphere temperature reconstruction ensemble ${ }^{5}$, this record reveals an extended cold period (1594-1677) in both hemispheres but no globally coherent warm phase during the pre-industrial (1000-1850) era. The current (post-1974) warm phase is the only period of the past millennium where both hemispheres are likely to have experienced contemporaneous warm extremes. Our analysis of inter-hemispheric temperature variability in an ensemble of climate model simulations for the past millennium suggests that models tend to overemphasize Northern Hemisphere-Southern Hemisphere synchronicity by underestimating the role of internal ocean-atmosphere dynamics, particularly in the ocean-dominated Southern Hemisphere. Our results imply that climate system predictability on decadal to century timescales may be lower than expected based on assessments of external climate forcing and Northern Hemisphere temperature variations $^{5,6}$ alone.

From over 25 hemispheric-scale temperature reconstructions published in recent decades, only three cover the ocean-dominated
Southern Hemisphere ${ }^{7}$. These Southern Hemisphere temperature reconstructions include only seven ${ }^{8}$ or fewer ${ }^{9}$ proxy datasets for the entire Southern Hemisphere, or were provided as peripheral components of Northern Hemisphere and global reconstruction efforts $^{4}$ with the caveat that 'more confident statements about long-term temperature variations in the Southern Hemisphere and globe on the whole must await additional proxy data collection' ${ }^{4}$. Consequently, attribution of temperature changes to external forcings ${ }^{10,11}$ and investigations of the coupling between temperature and greenhouse gas concentrations ${ }^{5,6}$ have focused on the Northern Hemisphere.

Data spanning inter-annual to multi-millennial timescales suggest limited temperature coherence between the two hemispheres. The degree of independence in Northern Hemisphere and Southern Hemisphere temperature trends over the past 150 years ${ }^{2}$ indicates that responses to external forcing may be modulated by ocean-atmosphere variability, reducing predictability of the climate system in twenty-first century model projections ${ }^{1,3}$. Patterns of late Quaternary deglaciation have also demonstrated high interhemispheric variability, attributed to a coupling of orbital forcing, ice-albedo feedbacks and the Atlantic Meridional Overturning Circulation ${ }^{12,13}$. Finally, a recent evaluation of multi-centennial reconstructions from seven continents also suggests stronger regional temperature coherence within the hemispheres than between them ${ }^{14}$. Yet, the preliminary nature of existing annually resolved Southern Hemisphere temperature reconstructions has hindered knowledge of the existence and driving mechanisms of inter-hemispheric climate variability on the societally relevant multi-decadal to centennial timescales.

Here, we introduce a Southern Hemisphere temperature reconstruction ensemble and assess inter-hemispheric temperature variability over the past millennium in both empirical reconstructions and state-of-the-art climate model simulations. We use an extensive Southern Hemisphere palaeoclimate data network from more than 300 individual sites ${ }^{15}$ yielding 111 temperature

\footnotetext{
${ }^{1}$ Oeschger Centre for Climate Change Research, University of Bern, CH-3012 Bern, Switzerland, ${ }^{2}$ Swiss Federal Institute WSL, Zürcherstrasse 111, CH-8903 Birmensdorf, Switzerland, ${ }^{3}$ School of Earth Sciences, University of Melbourne, Victoria 3010, Australia, ${ }^{4}$ ARC Centre of Excellence for Climate System Science, University of Melbourne, Victoria 3010, Australia, ${ }^{5}$ Department of the Environment, Australian Antarctic Division, Kingston, Tasmania 7050 , Australia, ${ }^{6}$ Antarctic Climate and Ecosystems Cooperative Research Centre, University of Tasmania, Hobart, Tasmania 7000, Australia, ${ }^{7}$ Departamento Astrofísica y CC de la Atmósfera, Instituto de Geociencias (CSIC-UCM) Universidad Complutense de Madrid, Madrid, 28040, Spain, ${ }^{8}$ Lamont-Doherty Earth Observatory, Columbia University, Palisades, New York 10964, USA, ${ }^{9}$ Instituto Argentino de Nivología, Glaciología y Ciencias Ambientales (IANIGLA), CCT CONICET-Mendoza, Mendoza, 5500, Argentina, ${ }^{10}$ LISEA, Facultad de Ciencias Agrarias y Forestales, Universidad Nacional de La Plata, B1900 La Plata, Argentina, ${ }^{11}$ Climate and Environmental Physics, University of Bern, CH-3012 Bern, Switzerland, ${ }^{12}$ Quaternary Research Center and Department of Earth and Space Sciences, University of Washington, Seattle, Washington 98195, USA, ${ }^{13}$ School of Earth and Environment, University of Western Australia Oceans Institute, 35 Stirling Highway, Crawley WA 6009, Australia, ${ }^{14}$ Australian Institute of Marine Science, Nedlands WA 6009, Australia. †Present address: University of Zürich, Physical Geography Division, Department of Geography, Winterthurerstrasse 190, CH-8057 Zürich, Switzerland. *e-mail: neukom@giub.unibe.ch
} 

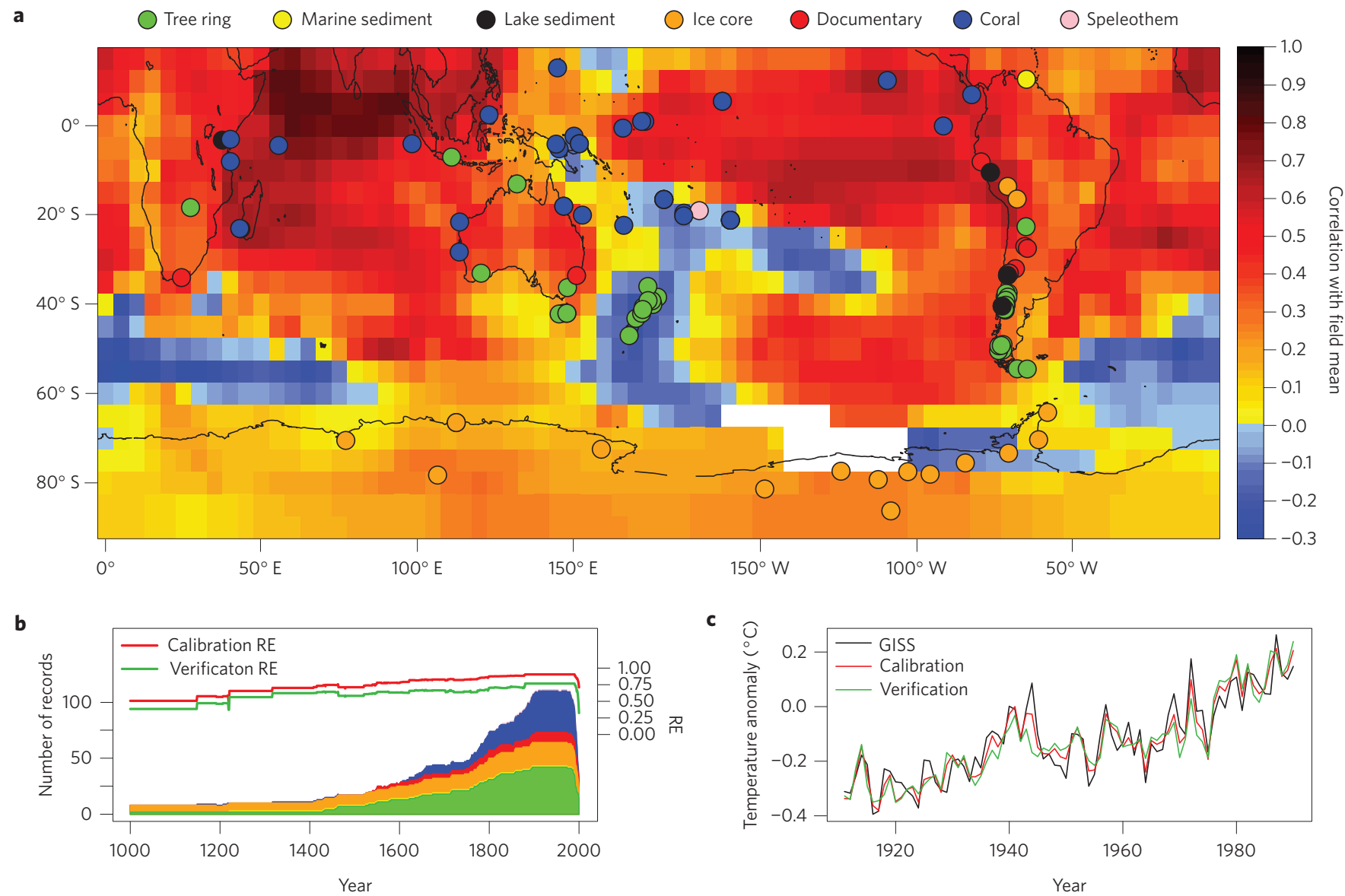

Figure 1 | Proxy data and calibration performance. a, Southern Hemisphere temperature reconstruction proxies. Shading represents GISS instrumental grid-cell temperature ${ }^{28}$ correlations in the period 1911-1990, with the Southern Hemisphere field mean used as reconstruction target (all data linearly detrended). Cells with less than 30 years of data are blank. b. Temporal evolution of the number of proxy time series used in the reconstruction, with colours indicating the relative contribution of each archive and calibration (red) and verification (green) RE skill metric for the period 1000-2000. c, Instrumental target temperatures (with respect to 1961-1990) over the 1911-1990 calibration/verification period (black) and reconstruction ensemble means of the years used for calibration (red) and verification (green) for the most replicated proxy nest. Details in Methods and Supplementary Section 3.1.3.

predictors (Supplementary Section 1). This proxy collection nearly doubles the number of records considered in the most advanced previous reconstruction attempt ${ }^{4}$, now allowing the development of an annually resolved and well-verified Southern Hemisphere temperature reconstruction for the past millennium (Fig. 1 and Supplementary Section 2) which is insensitive to moderate changes in reconstruction methodology or proxy network composition (Supplementary Section 3).

Although our database is weighted towards the Pacific sector of the Southern Hemisphere (Fig. 1a), the proxy network captures the inter-annual to long-term variability in Southern Hemisphere mean temperatures recorded by instrumental data (calibration: $r=0.73-0.95, \quad \mathrm{RE}=0.50-0.91$; verification: $r=0.57-0.88, \mathrm{RE}=0.32-0.77$; all $p \ll 0.01$; Fig. $1 \mathrm{~b}, \mathrm{c})$. The Southern Hemisphere reconstruction ensemble shows temperatures in the period 1000 to $1200 \mathrm{CE}$ (all years hereafter Common Era) that are close to the long term (1000-2000) average. This is followed by an approximately 150-year warm phase (1200-1350) containing the warmest pre-industrial temperatures of the past millenium (Fig. 2a). The subsequent long-term cooling trend reaches a minimum around 1600 , with negative decadal-scale ensemble-mean temperature anomalies prevailing until the early twentieth century. 99.7\% of the Southern Hemisphere reconstruction ensemble members indicate that the late twentieth century contained the warmest decade of the past millennium. This finding complements well-established evidence for the anomalous characteristics of Northern Hemisphere industrial-era warming ${ }^{5}$. Besides the positive twentieth century temperature anomalies, simultaneous cold anomalies in both hemispheres are identified between 1571 and 1722 (based on the 1000-2000 long term mean; Fig. 2a). During the rest of the millennium, the Northern Hemisphere and Southern Hemisphere are more prominently characterized by differences in the occurrence, timing and phase of warm and cold episodes. In medieval times, Southern Hemisphere temperature anomalies are notably colder than the Northern Hemisphere both before 1100 and around 1400, and warmer between 1280 and 1350. Expression of the industrial-era warming trend in the Southern Hemisphere also lags by approximately 25 years behind the Northern Hemisphere. Moreover, Southern Hemisphere temperatures tend to show a weaker cooling response to strong volcanic eruptions, for example, during the early nineteenth century.

To determine the extent to which reconstructed temperature patterns are independently identified by climate models, we investigate inter-hemispheric temperature coherence from a 24-member multi-model ensemble (simulation details in Supplementary Table 9). Very similar temperature evolutions are modelled for the two hemispheres (Fig. 2b). The majority of model ensemble members show warmest pre-industrial temperatures sometime between 1050 and 1250 in the Northern Hemisphere 
a

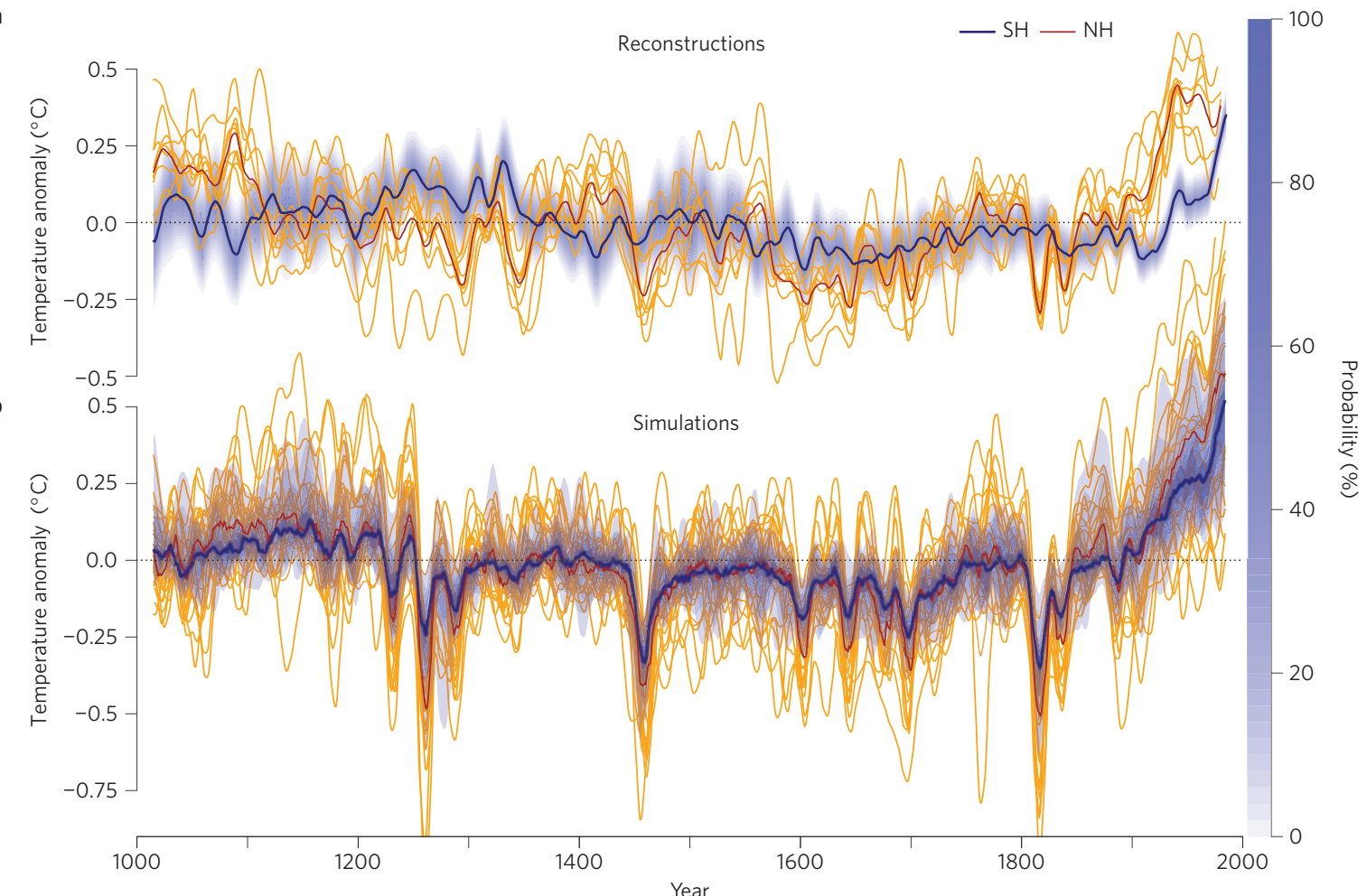

Figure 2 | Temperature variability over the past millennium. a, 30-year loess filtered ensemble mean temperature reconstruction for the Southern Hemisphere (SH; blue) and Northern Hemisphere ( $\mathrm{NH}$; red) relative to the millennium mean for the period 1000-2000. Blue shading based on Southern Hemisphere reconstruction uncertainties (Supplementary Section 2.4). Thin orange lines represent the ensemble means of the nine individual temperature reconstructions making up the Northern Hemisphere dataset ${ }^{5}$. b, as a but for the 24 -member climate model ensemble. Note for consistency with reconstruction data, simulated temperatures are shown as individual simulations for the Northern Hemisphere and a probabilistic range based on ensemble percentiles for the Southern Hemisphere.

(79\% of ensemble members), the Southern Hemisphere (75\%), and simultaneously in both $(67 \%)$ hemispheres. Interestingly, this simulated warm period is delayed compared to the reconstructed medieval warmth in the Northern Hemisphere and precedes the phase of maximum Southern Hemisphere pre-industrial warmth $^{16}$. Between 1300 and 1900, simulated temperatures are close to the 1000-2000 average, periodically interrupted by shorter volcanically induced cold excursions. In contrast to the delay in industrial Southern Hemisphere warming in the reconstructions, the climate model simulations show a mostly synchronous temperature increase after 1850. Mean correlations between 30-year filtered reconstructions and simulations for all possible ensemble pairs are $r=0.29 \pm 0.22$ ( $2 \sigma$ ensemble spread) for the Southern Hemisphere and $r=0.47 \pm 0.33$ for the Northern Hemisphere. These values increase to $r=0.35$ and $r=0.77$ for the ensemble means. As the model ensemble means are subjected less to internal variability than each individual simulation and better represent the temperature response to external forcing, these values suggest substantially weaker links between external forcing and Southern Hemisphere temperature variability compared to the Northern Hemisphere.

We quantify coherence between Northern Hemisphere and Southern Hemisphere temperature extremes by identifying the percentage of ensemble members showing decadal average temperatures more than one standard deviation above or below the 1000-2000 baseline (Fig. 3). Extended periods where at least $33 \%$ of the reconstruction ensemble members in both hemispheres simultaneously show extreme cold or warm temperatures are identified only between 1594 and 1677 and since 1967, respectively. Since 1974 more than 66\%, and since 1979 more than 90\%, of ensemble members show synchronous positive extremes (corresponding to 'likely' and 'very likely' categories using IPCC AR5 calibrated uncertainty classification). This analysis provides evidence for a global cold phase coinciding with the peak of the Northern Hemisphere 'Little Ice Age' (LIA) and a late-twentieth century warm phase of unprecedented duration and magnitude within the past 1000 years. In contrast, we find no empirical support for a globally coherent 'Medieval Climate Anomaly' (MCA) at decadal timescales during the past millennium ${ }^{14}$. Our new temperature reconstruction from the Southern Hemisphere suggests that data from the Northern Hemisphere alone are insufficient to characterize global scale temperature anomalies, trends and extremes.

Simulated extreme conditions shown in Fig. 3 are a direct expression of external forcing (Fig. 3d). The notable reconstructed seventeenth-century peak LIA is less prominent in the model ensemble, which shows the clearest global cooling signal in response to volcanic eruptions around 1815 (Tambora), the 1450s (Kuwae) and 1258 (Samalas). External climate forcing as incorporated in the current climate model simulations does not account for key features of reconstructed temperature variation. This suggests that internal variability was a key driver for hemispheric and global decadal-scale extreme periods.

To further investigate inter-hemispheric temperature coherence, we calculate the ten-year running temperature differences between the standardized Northern Hemisphere and Southern Hemisphere reconstructions (Fig. 4a). The variability and amplitude of the Northern Hemisphere-Southern Hemisphere temperature difference fluctuates considerably over time, showing periods with large divergence (for example, around 1100 and 1575) and with more in-phase variability (for example, the thirteenth and eighteenth centuries). The distinct and internally driven 


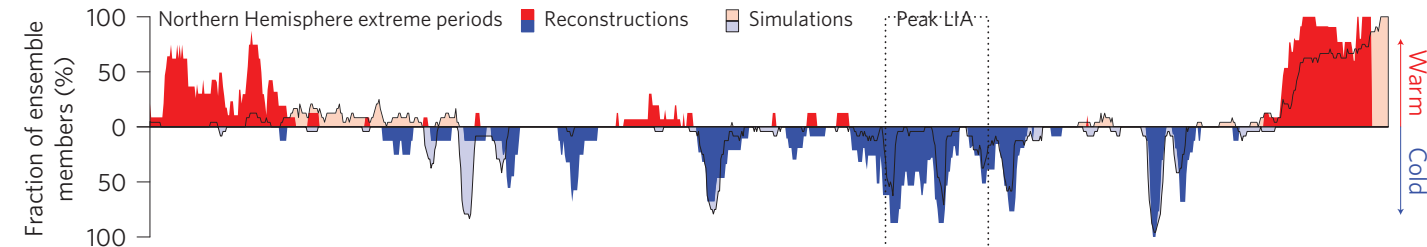

b



c

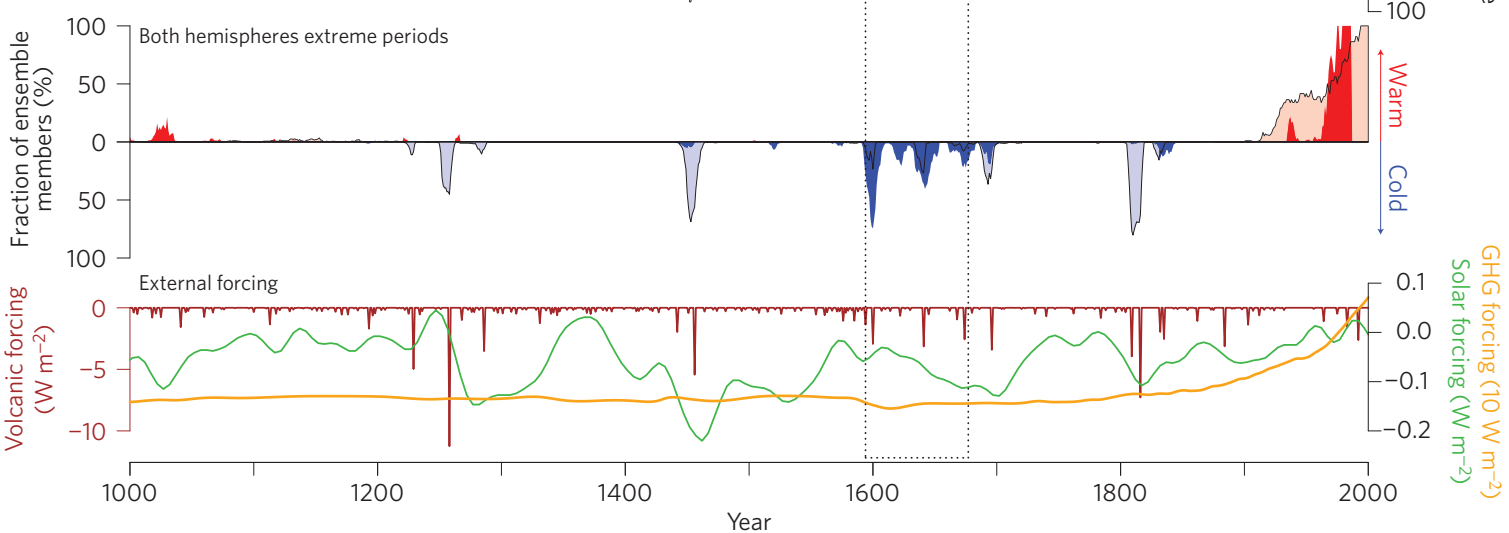

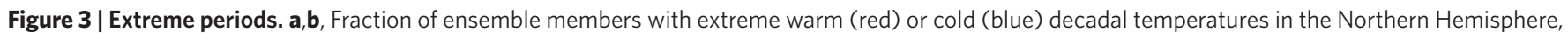
(a) and Southern Hemisphere (b), respectively. Dark shading represents the reconstructions, light shading with black border the model simulations. c, Probabilities for simultaneous extreme periods in both hemispheres are calculated by multiplying the fractions in $\mathbf{a}$ and $\mathbf{b}$. $\mathbf{d}$, Volcanic ${ }^{29}$ (brown), solar ${ }^{30}$ (green) and greenhouse gas forcing ${ }^{19}$ (yellow) relative to 1961-1990. Dotted lines enclose the globally expressed peak Little Ice Age (LIA) 1594-1677.

drop in Northern Hemisphere temperatures around $1970^{2}$ was preceded by several other analogous periods of contrasting hemispheric temperature trends. Internal ocean-atmosphere processes appear to be the main driver of the larger Northern Hemisphere-Southern Hemisphere differences; only two episodes of contrasting temperature regimes coincide with strong volcanic eruptions (Kuwae and Tambora). Model simulations also contain periods of contrasting inter-hemispheric temperature trends, but with notably smaller differences between the hemispheres (Supplementary Figs 36-59): median reconstructed Northern Hemisphere-Southern Hemisphere differences are outside the 10th-90th percentile range of model simulations $42 \%$ of the time (Fig. 4). The lower Northern Hemisphere-Southern Hemisphere temperature contrasts within the simulations are not only evident in the pre-instrumental period but also during the twentieth century ${ }^{3}$ (Fig. 4b,c), when both the reconstructions and instrumental data ${ }^{2}$ show strong inter-hemispheric variability.

The Southern Hemisphere reconstruction presented here allows new insights into the characteristics of the global climate system. For example, it has been proposed that the Southern Hemisphere response to external forcing may be delayed and buffered by the large heat capacity of the oceans ${ }^{17,18}$. The greater amplitude of pre-industrial temperature variation in the Northern Hemisphere $\left(0.67{ }^{\circ} \mathrm{C} \pm 0.46^{\circ} \mathrm{C}(2 \sigma\right.$ ensemble spread $)$ versus $0.37^{\circ} \mathrm{C} \pm 0.11^{\circ} \mathrm{C}$ in the Southern Hemisphere), the approximately two-century Northern Hemisphere lead during medieval times and the approximately 25 year lead during the era of industrial warming are in line with this hypothesis. However, we find no evidence for a consistent lag between Northern Hemisphere and Southern Hemisphere temperatures (Supplementary Section 8). The coherent and extreme cool conditions in both hemispheres around 1600 are unique within the past millennium and now offer perhaps the most viable explanation for the drop in global $\mathrm{CO}_{2}$ (difference of $8.37 \mathrm{ppm}$ or $0.19 \mathrm{~W} \mathrm{~m}^{-2}$ between $1540-1580$ and $1600-1640^{5,19,20}$; Fig. 3d), which may not be sufficiently explained by land use change ${ }^{21}$ or Northern Hemisphere-temperature$\mathrm{CO}_{2}$ feedbacks ${ }^{5}$.

Our results suggest that large, internally driven temperature contrasts between the hemispheres, such as identified in the twentieth century ${ }^{2}$ have repeatedly occurred on the policyrelevant multi-decadal to centennial timescales. This finding is strengthened by evidence from annually resolved regional temperature reconstructions ${ }^{14,22,23}$ and the timing of glacial fluctuations in New Zealand and the Northern Hemisphere ${ }^{24}$. Our data support hypotheses that global and hemispheric temperature extremes and transitions may be initiated ${ }^{11,16,25}$ and prolonged ${ }^{26}$ by internal variability and feedbacks. Analyses targeting periods where climate models and reconstructions differ will be necessary to identify weaknesses in both proxy- and model-based representations of the Earth's climate system. However, the strong inter-hemispheric coupling in the simulations assessed herein suggests that models overestimate the strength of externally forced relative to internal climate system variability, therefore implying more limited predictability not only on regional ${ }^{1,27}$ but also hemispheric scales. The stronger coherence between the Northern Hemisphere temperature reconstructions and external forcings similarly implies that detection and attribution studies ${ }^{10}$ and climate sensitivity estimates ${ }^{5,6}$ based on Northern Hemisphere data alone may not be representative of the global climate system. Future consideration of Southern Hemisphere temperature evolution should reduce uncertainties in estimating and attributing natural and anthropogenically forced climate variations. 
a

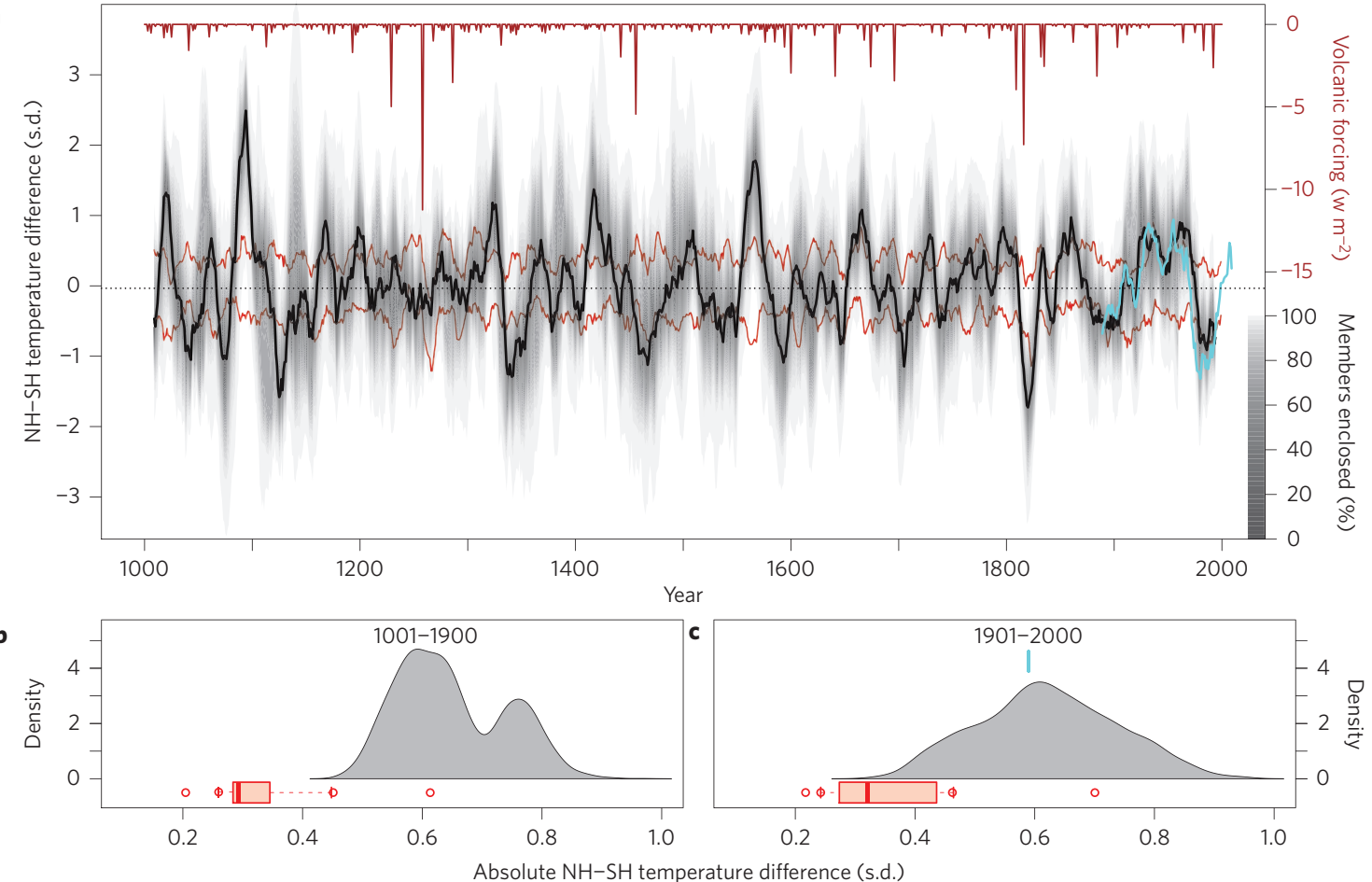

Figure 4 | Inter-hemispheric temperature difference. a, Difference between 10-year filtered Northern Hemisphere-Southern Hemisphere temperatures in the ensemble reconstructions after detrending and standardizing (black). The solid line represents the ensemble median, grey shading the percentiles. Cyan shows the instrumental data 1880-201028. Red lines are the 10th and 90th percentiles from the ensemble of model simulations. Volcanic forcing ${ }^{29}$ shown at the top in brown. b. Ensemble distribution of the reconstructed absolute Northern Hemisphere-Southern Hemisphere temperature difference averaged over the 1000-1900 period. Red boxplot represents model simulations. c as b but for the twentieth century, cyan represents instrumental data.

\section{Methods}

Southern Hemisphere reconstruction ensemble. We use the Southern Hemisphere spatial mean of the Goddard Institute for Space Studies (GISS) Surface Temperature Analysis (GISTEMP) temperature grid $^{28}$ as the instrumental predictand for the reconstruction. The palaeoclimate data network ${ }^{15}$ consists of 48 marine (46 coral and 2 sediment time series) and 277 terrestrial (206 tree-ring sites, 42 ice core, 19 documentary, 8 lake sediment and 2 speleothem) records (details in Supplementary Section 1). Although proxy records are preferentially located towards land areas, the network represents a considerable improvement of both geographical coverage and proxy quantity and quality (for example, resolution, length) since the last Southern Hemisphere reconstruction effort ${ }^{4}$. Proxies are screened with local grid-cell temperatures ${ }^{28}$ yielding 111 temperature predictors (Fig. 1) for the nested multivariate principal component regression procedure $^{23}$. A 3,000-member ensemble reconstruction of annual Southern Hemisphere temperatures over the period 1000-2000 was generated with the spread of ensemble members considered a measure of uncertainty.

For each ensemble member we use different reconstruction parameters by randomly selecting a subset of proxies, as well as varying the calibration/verification intervals within 1911-1990, and other reconstruction parameters (details in Supplementary Section 2.2). The perturbation of calibration/verification periods allows a 'verification ensemble mean' to be calculated over the 1911-1990 period by averaging all members where a given year was used for verification (and not for calibration). Analogously, a 'calibration ensemble mean' was calculated. These time series and their corresponding Reduction of Error (RE) skills are shown in Fig. 1c,b, respectively. These statistics along with additional verification based on the sparse early Southern Hemisphere instrumental data $(\mathrm{RE}=0.41-0.90$; Supplementary Fig. 10) point to reconstructive skill over the past millennium. In addition to traditional reconstruction uncertainty estimates based on regression residuals, we assess the influence of the ensemble perturbations on the reconstruction outcome. Uncertainty envelopes in Fig. 2a represent combined calibration and ensemble uncertainties (details in Supplementary Section 2.4).

Although we have taken steps to provide robust results considering the challenges of proxy-based reconstructions (for example, potential underestimation of past climate amplitudes) discussed in the literature, we note that all reconstruction approaches contain uncertainties. The fact that our reconstruction verifies well and captures interannual and decadal-scale temperature fluctuations during the instrumental period (Fig. 1 and
Supplementary Section 3) indicates reduced probability of such artefacts. An extensive assessment of reconstruction robustness is provided in Supplementary Section 3.2 and Supplementary Figs 13-26, with tests demonstrating that the potential bias introduced by the proxy-screening and reconstruction methods or by single dominant records or proxy archives is small.

Northern Hemisphere reconstruction ensemble. Details concerning the Northern Hemisphere reconstructions are provided in ref. 5 and Supplementary Section 5. The most important difference from our Southern Hemisphere reconstruction is that it is not based on a single predictor matrix but uses nine published Northern Hemisphere reconstructions based on different (but not independent) proxy sets and various reconstruction methodologies. In ref. 5, the individual single-member reconstructions were recalibrated to instrumental temperature data using different calibration periods as ensemble parameters, resulting in a total of 521 ensemble members. The Northern Hemisphere ensemble spread is larger than in the Southern Hemisphere owing to the relatively large differences between some of the original sub-reconstructions and the composite-plus-scaling approach over a range of time windows in ref. 5 . To best illustrate these two approaches, the ensemble means of the nine sub-reconstructions are shown for the Northern Hemisphere in Fig. 2a. As a consequence of these methodological differences and the larger ensemble spread in the Northern Hemisphere, one would expect generally reduced probabilities for extreme periods in the Northern Hemisphere. However, Fig. 3a,b shows similar fractions of periods with high probabilities for extremes, indicating a similar consistency between ensemble members in the timing of extreme periods in both hemispheres.

Extreme periods (Fig. 3). To quantify extreme periods, we calculate 10-year running averages relative to the 1000-2000 mean. All years exceeding the \pm 1 standard deviation range (calculated over the unfiltered 1000-2000 period) are considered extreme. We quantify the probability of extreme periods as the fraction of ensemble members exceeding this threshold for each year (Fig. 3a,b). Probabilities for simultaneous extreme periods are calculated by multiplying the probabilities of each hemisphere (Fig. 3c).

Northern Hemisphere-Southern Hemisphere difference (Fig. 4). To evaluate the decadal to centennial coherence, reconstruction and climate model data are detrended using a 200-year loess-filter before the analysis. Instrumental data are 
linearly detrended. Ten-year running averages are then calculated and divided by the standard deviation over 1000-2000 to allow relative comparison of hemispheric fluctuations. Each of the standardized filtered Southern Hemisphere reconstructions is subtracted from a randomly selected Northern Hemisphere reconstruction, with shaded probabilities in Fig. 4a indicating the fraction of ensemble members enclosed. Modelled Northern Hemisphere-Southern Hemisphere differences are calculated individually for each simulation (alternative calculations in Supplementary Section 10). The distributions in Fig. $4 \mathrm{~b}, \mathrm{c}$ are calculated from the average absolute Northern

Hemisphere-Southern Hemisphere difference over the respective time interval for each ensemble member. Boxes, whiskers and circles of the boxplots in Fig. 4b,c represent interquartile range, 5 th/95th percentiles and extremes, respectively; bold line indicates the median.

Received 18 November 2013; accepted 25 February 2014; published online 30 March 2014

\section{References}

1. Deser, C., Knutti, R., Solomon, S. \& Phillips, A. S. Communication of the role of natural variability in future North American climate. Nature Clim. Change 2, 775-779 (2012)

2. Thompson, D. W. J., Wallace, J. M., Kennedy, J. J. \& Jones, P. D. An abrupt drop in Northern Hemisphere sea surface temperature around 1970. Nature 467, 444-447 (2010)

3. Friedmann, A., Hwang, Y., Chiang, J. \& Frierson, D. Interhemispheric temperature asymmetry over the 20th century and in future projections. J. Clim. 26, 5419-5433 (2013).

4. Mann, M. et al. Proxy-based reconstructions of hemispheric and global surface temperature variations over the past two millennia. Proc. Natl Acad. Sci. USA 105, 13252-13257 (2008).

5. Frank, D. C. et al. Ensemble reconstruction constraints on the global carbon cycle sensitivity to climate. Nature 463, 527-532 (2010).

6. Hegerl, G. C., Crowley, T. J., Hyde, W. T. \& Frame, D. J. Climate sensitivity constrained by temperature reconstructions over the past seven centuries. Nature 440, 1029-1032 (2006).

7. Frank, D., Esper, J., Zorita, E. \& Wilson, R. A noodle, hockey stick, and spaghetti plate: A perspective on high-resolution paleoclimatology. WIREs Clim. Change 1, 507-516 (2010).

8. Jones, P. D., Briffa, K. R., Barnett, T. P. \& Tett, S. F. B. High-resolution palaeoclimatic records for the last millennium: Interpretation, integration and comparison with General Circulation Model control-run temperatures. Holocene 8, 455-471 (1998).

9. Mann, M. E. \& Jones, P. D. Global surface temperatures over the past two millennia. Geophys. Res. Lett. 30, 1820 (2003).

10. Hegerl, G. et al. Detection of human influence on a new, validated 1500-year temperature reconstruction. J. Clim. 20, 650-666 (2007).

11. Goosse, H. et al. The role of forcing and internal dynamics in explaining the "Medieval Climate Anomaly". Clim. Dynam. 39, 2847-2866 (2012).

12. WAIS Divide Project Members. Onset of deglacial warming in West Antarctica driven by local orbital forcing. Nature 500, 440-444 (2013).

13. Shakun, J. D. et al. Global warming preceded by increasing carbon dioxide concentrations during the last deglaciation. Nature 484, 49-54 (2012)

14. PAGES $2 \mathrm{k}$ Consortium. Continental-scale temperature variability during the past two millennia. Nature Geosci. 6, 339-346 (2013).

15. Neukom, R. \& Gergis, J. Southern Hemisphere high-resolution palaeoclimate records of the last 2000 years. Holocene 22, 501-524 (2012).
16. Fernández-Donado, L. et al. Large-scale temperature response to external forcing in simulations and reconstructions of the last millennium. Clim. Past 9, 393-421 (2013).

17. Stouffer, R. J., Manabe, S. \& Bryan, K. Interhemispheric asymmetry in climate response to a gradual increase of atmospheric $\mathrm{CO}_{2}$. Nature 342, 660-662 (1989)

18. Goosse, H. et al. A late medieval warm period in the Southern Ocean as a delayed response to external forcing? Geophys. Res. Lett. 31, L06203 (2004).

19. MacFarling-Meure, C. et al. Law Dome $\mathrm{CO}_{2}, \mathrm{CH}_{4}$ and $\mathrm{N}_{2} \mathrm{O}$ ice core records extended to 2000 years BP. Geophys. Res. Lett. 33, L14810 (2006).

20. Jungclaus, J. H. et al. Climate and carbon-cycle variability over the last millennium. Clim. Past 6, 723-737 (2010).

21. Pongratz, J., Caldeira, K., Reick, C. H. \& Claussen, M. Coupled climate-carbon simulations indicate minor global effects of wars and epidemics on atmospheric $\mathrm{CO}_{2}$ between $\mathrm{AD} 800$ and 1850. Holocene 21, 843-851 (2011).

22. Wahl, E. R. \& Smerdon, J. E. Comparative performance of paleoclimate field and index reconstructions derived from climate proxies and noise-only predictors. Geophys. Res. Lett. 39, L06703 (2012).

23. Neukom, R. et al. Multiproxy summer and winter surface air temperature field reconstructions for southern South America covering the past centuries. Clim. Dynam. 37, 35-51 (2011).

24. Schaefer, J. M. et al. High-frequency holocene glacier fluctuations in New Zealand differ from the northern signature. Science 324, 622-625 (2009).

25. González-Rouco, F. J. et al. Medieval Climate Anomaly to Little Ice Age transition as simulated by current climate models. PAGES News 19, 7-8 (2011).

26. Miller, G. H. et al. Abrupt onset of the Little Ice Age triggered by volcanism and sustained by sea-ice/ocean feedbacks. Geophys. Res. Lett. 39, L02708 (2012)

27. Braconnot, P. et al. Evaluation of climate models using palaeoclimatic data. Nature Clim. Change 2, 417-424 (2012).

28. Hansen, J., Ruedy, R., Sato, M. \& Lo, K. Global surface temperature change. Rev. Geophys. 48, RG4004 (2010)

29. Crowley, T. J. et al. Volcanism and the Little Ice Age. PAGES News 16, 22-23 (2008).

30. Steinhilber, F., Beer, J. \& Fröhlich, C. Total solar irradiance during the Holocene. Geophys. Res. Lett. 36, L19704 (2009).

\section{Acknowledgements}

This work has been possible thanks to the collaboration of many members of the Australasian, South American, African and Antarctic working groups of the PAGES Regional $2 \mathrm{~K}$ initiative. Researchers from these working groups are warmly thanked for providing metadata inventories and access to data. S. J. Phipps is acknowledged for support with model data. We acknowledge funding from the Swiss National Science Foundation and funding from the Australian Research Council (Projects LP0990151 and DE130100668) and the Australian Department of Climate Change and Energy Efficiency.

\section{Author contributions}

R.N. performed data analyses and developed the figures and tables. R.N., D.F. and J.G. led the writing of the paper. R.N., J.G., D.J.K., H.W. and D.F. designed the study. M.C., J.E. B.K.L, A.D.M., I.M., E.J.S., T.V., R.V., T.v.O. and J.Z. assisted with proxy data and F.G. and C.C.R. with climate model data. All authors discussed the results and commented jointly on the manuscript.

\section{Additional information}

Supplementary information is available in the online version of the paper. Reprints and permissions information is available online at www.nature.com/reprints. Correspondence and requests for materials should be addressed to R.N.

\section{Competing financial interests}

The authors declare no competing financial interests. 